\title{
Análisis de los sistemas de flujo en un acuífero perturbado por la extracción de aguas subterráneas. Caso zona Morelia-Capula, Michoacán
}

\author{
José Pérez-Villarreal, Jorge Alejandro Ávila-Olivera, Isabel Israde-Alcántara
}

José Pérez-Villarreal

Jorge Alejandro Ávila-Olivera

ja.avilaolivera@gmail.com

Instituto de Investigaciones sobre los Recursos Naturales (INIRENA) de la Universidad Michoacana de San Nicolás de Hidalgo (UMSNH). Avenida Juanito Itzícuaro S/N, Nueva Esperanza, G.P. 58330, Morelia, Michoacán, México.

\section{Isabel Israde-Alcántara}

Instituto de Investigaciones en Ciencias de la Tierra (INICIT) de la Universidad Michoacana de San Nicolás de Hidalgo (UMSNH). Avenida FranciscoJ. Múgica S/N, Edificio U-4 Ciudad Universitaria, C.P. 58030, Morelia, Michoacán, México.

\section{José Pérez-Villarreal}

Instituto Tecnológico Superior de Uruapan. Carretera Uruapan-Carapan \#5555, La Basilia, C.P. 60015, Uruapan, Michoacán, México.

BOL. SOC. GEOL. MEX. 2018

VOL. 70 NO. 3

P. $675-688$

http://dx.doi.org/10.18268/BSGM2018v70n3a5

\section{RESUMEN}

La zona Morelia-Capula depende totalmente de agua subterránea para el abasto de sus más de 120000 habitantes. La extracción del agua subterránea ha ocasionado que en los pozos el nivel piezométrico se haya abatido más de 45 metros en un periodo de 21 años. Dada la importancia del sistema acuífero de la zona Morelia-Capula es necesario profundizar en su entendimiento, por lo cual se aborda su estudio desde la perspectiva de los sistemas de flujo Tóthianos. Se recurre a tres parámetros para identificar la dinámica del agua subterránea, la temperatura, los iones mayores y la profundidad (nivel estático). Se analizaron muestras de agua subterránea en un total de 35 sitios (29 pozos y 6 manantiales), se encontraron 26 flujos intermedios, 8 locales y 1 regional. El sistema de flujo local se ubica en los estratos superiores del sistema acuífero y se evidencia en pozos poco profundos y manantiales. El sistema de flujo intermedio se encuentra en toda la zona de estudio por debajo de los locales; la mayor parte de los pozos están extrayendo agua de este sistema. Los sistemas de flujo local e intermedio se localizan tanto en la unidad terrígena como en la volcánica. El sistema de flujo regional se encuentra únicamente en la unidad volcánica que subyace a la terrígena, y pocos pozos han entrado en contacto con este sistema de flujo; la tendencia del abatimiento muestra que en los próximos años este sistema será el que provea de agua a la zona. Se encontró que la extracción intensiva y la presencia de fallas geológicas favorecen la mezcla de flujos. La mayor parte del abasto hídrico de la zona depende del sistema de flujo intermedio. La zona presenta un abatimiento anual promedio de $2.13 \mathrm{~m} /$ año. La dirección de flujo de los flujos locales e intermedios sigue en general la topografía de la zona, aunque se presentan alteraciones puntuales debido a la formación de conos de abatimiento. Los resultados indican que es necesario implementar estrategias para evitar contaminar los flujos locales y por otra parte disminuir la presión hídrica derivada de la extracción en los sistemas intermedio y regional.

Palabras clave: Sistemas de flujo, agua subterránea, temperatura, iones mayores, abatimiento, zona Morelia-Capula.

\section{ABSTRACT}

The Morelia-Capula zone depends entirely on groundwater for the supply of its more than 120000 inhabitants. The groundwater extraction has caused a decrease in the piezometric level estimated at more than 45 meters in a period of 21 years. Because of the importance of the aquifer system of the MoreliaCapula zone it is necessary to have a complete understating of the system, therefore, this study is carried out from the perspective of the Tothian flow systems. Three parameters are used to identify the groundwater dynamics, temperature, major ions, and depth (static level). Groundwater samples were analyzed in a total of 35 sites (29 wells and 6 springs), consisting of 26 intermediate, 8 local, and 1 regional flows. The local flow system is located in the upper strata of the aquifer system and can be seen within the shallow wells and springs. The intermediate flow system is located throughout the study area below the local flow system where most wells are extracting water from this system. The local and intermediate flow systems are localized in the terrigenous and volcanic units. The regional flow system is only in the volcanic unit that underlies the terrigenous unit. A few wells have come in contact with this system. A depletion trend shows that in the next few years this flow system will provide water to this zone. It was found that intensive extraction and the presence of geological faults favor the mixture of flows. Most of the water supply in the zone depends on the intermediate flow system. The static level in the zone descends $2.13 \mathrm{~m} /$ year. The flow direction of local and intermediate systems generally follows the topography of the zone, although there are local alterations due to the formation of depletion cones. Results indicate the need to implement strategies to avoid contaminating local flows and to reduce the water pressure derived from extraction toward the intermediate and regional systems.

Keywords: Flow systems, groundwater, temperature, major ions, depletion, Morelia-Capula zone. 


\section{Introducción}

En México el agua subterránea cobra cada día mayor importancia. Las estadísticas oficiales señalan que en el año 2014 del total de agua para usos consuntivos el $38.70 \%$ fue de origen subterráneo (CONAGUA, 2015). El agua subterránea es particularmente importante en el contexto del suministro urbano (Dávila-Pórcel y de León-Gómez, 2011). Varias de las grandes ciudades de México dependen casi por completo del agua subterránea para satisfacer su necesidad hídrica, tal es el caso de ciudades como Querétaro, San Luis Potosí y Aguascalientes. La extracción continua de agua subterránea presenta potenciales riesgos, como los acelerados abatimientos de los niveles piezométricos (Ávila-Olivera y Garduño-Monroy, 2007) y el detrimento de la calidad del agua subterránea extraída de pozos (Cardona y Carrillo-Rivera, 2006; Huizar-Alvarez et al., 2004), ambos fenómenos directamente relacionados con la dinámica del agua subterránea. Existen diferentes enfoques para conocer el comportamiento del agua subterránea en una determinada zona de estudio. En este contexto, la teoría de sistemas de flujo propuesta por Tóth (1963) permite reconocer la dinámica del agua subterránea integrando los elementos del medio geológico, suelo y vegetación original y el factor humano. Por lo tanto, este enfoque es cada vez más recurrido para entender los fenómenos de respuesta ambiental entorno al agua subterránea (Peñuela-Arévalo y Carrillo-Rivera, 2013). Tóth (1963) clasifica los flujos en locales, intermedios y regionales; las características que describen a los diferentes sistemas de flujo se detallan en la literatura (Carrillo-Rivera y Cardona, 2012; Tóth, 1963). La presente investigación estudia una zona ubicada al poniente de Morelia, Michoacán, donde hay una extracción intensiva del agua subterránea, que es la única fuente de abastecimiento, lo cual ha generado abatimientos de los niveles piezométricos, que en casos drásticos ha llevado a que algunos pozos se clausuren o requieran ser reperforados a mayor profundidad. Con el objetivo de entender la problemática desde una visión integral, se analiza el sistema acuífero desde la perspectiva de la teoría de sistemas de flujo, y a partir de ella se reconoce la dinámica del agua subterránea y las potenciales implicaciones en su manejo.

\section{Descripción de la zona de estudio}

La zona Morelia-Capula pertenece a la zona hidrográfica Lerma-Chapala, dentro de la cuenca del Lago de Cuitzeo, en una subcuenca de tipo exorreico denominada Morelia-Capula. El clima de la zona se clasifica como templado con lluvias en verano, concentrándose éstas en los meses de julio y agosto (Carlón-Allende y Mendoza, 2007). Los registros indican que la precipitación media anual es de $707.9 \mathrm{~mm}$ y la temperatura media anual es de $17.20^{\circ} \mathrm{C}$ (mínima de $7.40^{\circ} \mathrm{C}$ y máxima de $22.90^{\circ} \mathrm{C}$ ).

Desde el punto de vista geológico, la zona de estudio de Morelia-Capula se encuentra dentro de la provincia geológica denominada Faja Volcánica Trans-Mexicana (FVTM); desde la perspectiva geomorfológica se ubica en un paisaje compuesto por lomas y valles localizado entre importantes cuerpos volcánicos, al noreste por el Quinceo y Las Tetillas (ambos de tipo escudo) y al suroeste por el cerro del Águila (semiescudo) (GarduñoMonroy et al., 2010). La zona ha tenido actividad volcánica reciente, en los últimos 10000 años hay registro de reactivaciones del Quinceo (GarduñoMonroy et al., 2014). En la mayor parte de la superficie de la zona de estudio aflora material volcánico fracturado (principalmente basaltos), además hay presencia de arenas y cenizas. $\mathrm{Al}$ este de la zona de estudio se encuentra un área caracterizada por la presencia de material lacustre (arcilla) y fluviolacustre (arenas, conglomerados y gravas), evidenciando la existencia de un paleolago del cual quedan rastros, ya que aún se encuentran sitios de inundación que aparecen durante la época de lluvias formando un cuerpo de agua 
superficial de menos de un metro de profundidad. En la Figura 1 se presenta la distribución de los materiales geológicos y la ubicación de los sitios de estudio. Otro elemento que añade complejidad al acuífero es la presencia de fallas geológicas, las cuales pueden actuar como fronteras de flujo impidiendo o facilitando la interconexión hidráulica de acuíferos (Carreón-Freyre et al., 2005; Ochoa-
González et al., 2015). Las fallas geológicas de la zona Morelia-Capula tienen dirección este-oeste y están alineadas con el sistema regional MoreliaAcambay (Israde-Alcántara et al., 2004). La falla de la Paloma (perteneciente al sistema MoreliaAcambay) delimita el sur de la zona de estudio, y en ella se encuentran manifestaciones termales como el manantial Cointzio.
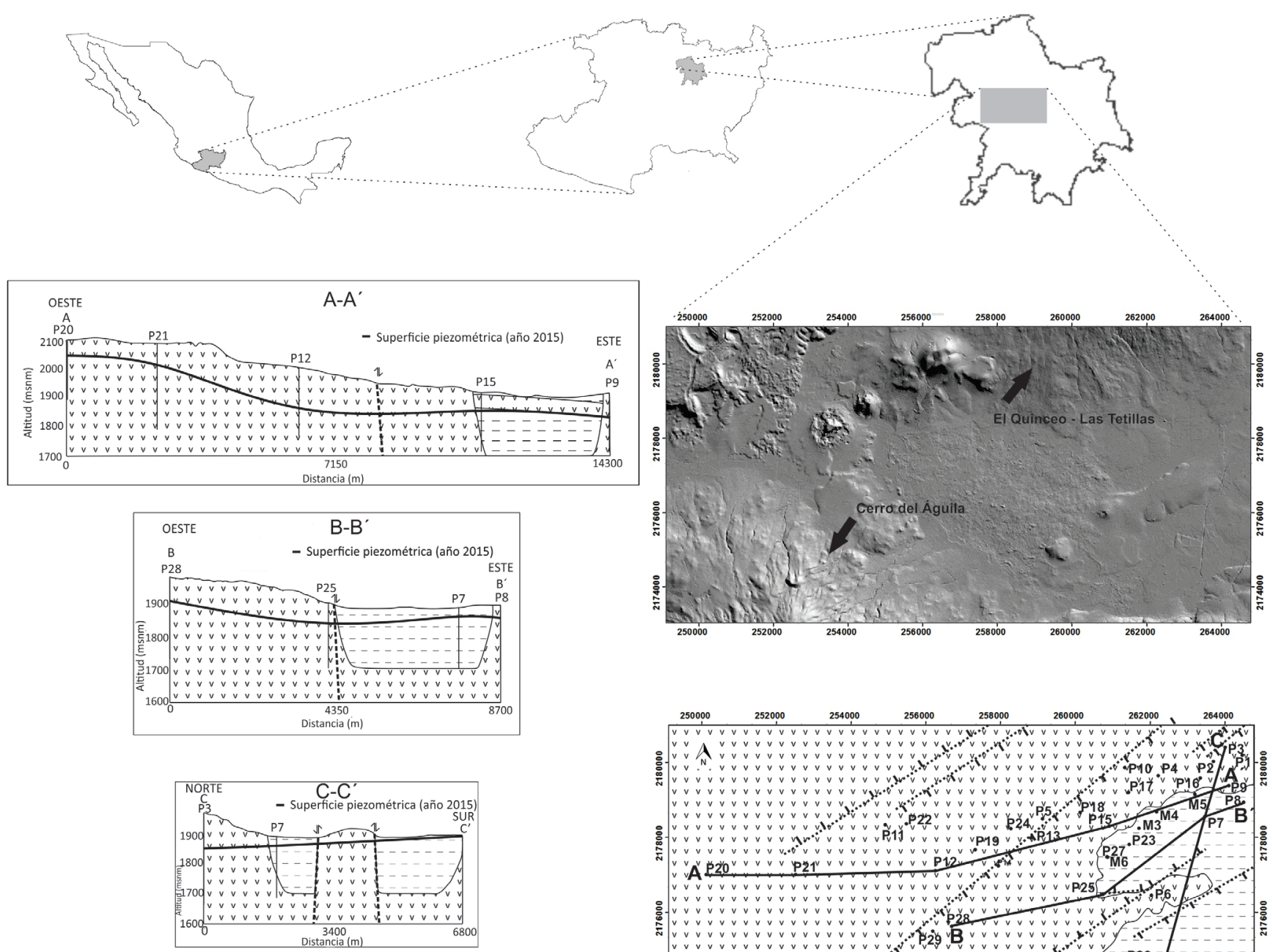

Simbología

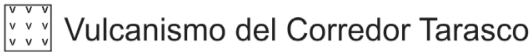

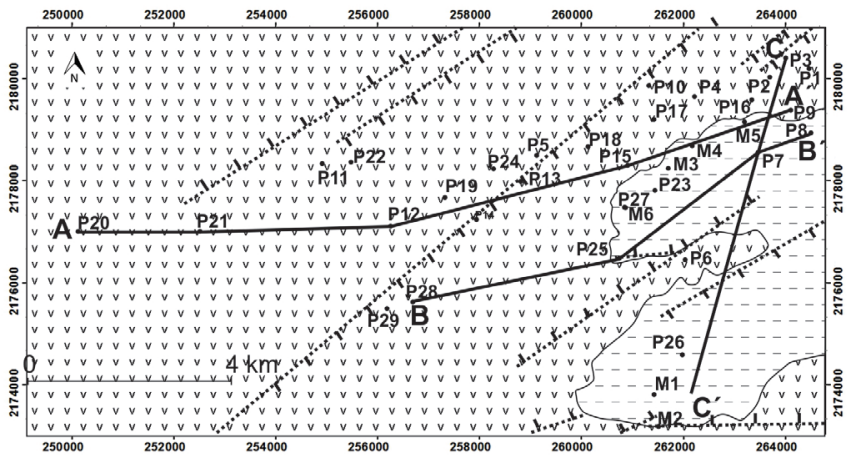

Secuencias Lacustres y Fluviolacustres

- Fallas geológicas

- Sitio de estudio (pozo o manantial)

Figura 1 Mapa mostrando la subcuenca de Morelia-Capula ubicada en el sector norte-centro del Estado de Michoacán y las secciones geológicas realizadas en base a la altitud y la información litológica de las unidades atravesadas por los pozos. 
La zona de estudio ha experimentado un crecimiento poblacional de unos 20000 habitantes en el año 1994 a más de 120000 en el 2015, ocasionado un incremento de la extracción de agua subterránea que conlleva a un abatimiento acelerado de la superficie piezométrica. Los registros del Organismo Operador de Agua Potable, Alcantarillado y Saneamiento (OOAPAS), encargado de la administración del agua a nivel municipal, señalan que en el año 1994 el nivel estático de la zona se encontraba a $30 \mathrm{~m}$ de profundidad; en la actualidad se detectaron pozos cuyo nivel estático supera los $100 \mathrm{~m}$. La zona Morelia-Capula se ubica en un área de transición entre un medio urbano y un medio rural. Básicamente, toda el agua que se consume en la zona de estudio se destina al uso doméstico, aunque existen sitios de cultivo que son de temporal. Se considera a la zona MoreliaCapula como un lugar de recarga para el sistema acuífero, ya que su suelo de origen volcánico es permeable y recibe escurrimientos que provienen de los volcanes las Tetillas, el Quinceo y el cerro del Águila (Garduño-Monroy et al., 2014). El flujo de agua subterránea en el plano horizontal va de oeste a este, siguiendo la topografía de la zona (Israde-Alcántara et al., 2005), que está asociada a la presencia de fallas geológicas, las cuales pueden actuar como fronteras de flujo, impidiendo o facilitando la interconexión de acuíferos (CarreónFreyre et al., 2005; Ochoa-González et al., 2015). La interpretación de los registros litológicos de las perforaciones y los datos obtenidos de los sondeos eléctricos verticales permiten identificar que en los primeros $400 \mathrm{~m}$ de profundidad de la zona Morelia-Capula existe un sistema acuífero compuesto por dos unidades hidrogeológicas, una volcánica y le sobreyace otra terrígena de origen lacustre y fluviolacustre. La unidad volcánica es producto de la actividad efusiva principalmente del corredor Tarasco que incluye al norte a los volcanes semiescudo Quinceo y Tetillas cuyas emisiones han sido fechadas en 1.3 millones de años (Cisneros-Máximo, 2016), el Quinceo ha tenido distintos periodos eruptivos, el último al inicio del Holoceno. Se desconoce el espesor de la unidad volcánica ya que los cortes litológicos realizados en la zona no han atravesado esta unidad. La unidad terrígena conforma un antiguo paleolago y presenta un espesor aproximado que varía de entre 200 y 250 m. Los sedimentos lacustres y fluviolacustres están constituidos por arenas y arcillas. Por medio de la geología regional se ha determinado que el basamento del sistema acuífero es el complejo andesítico de Mil Cumbres, el cual ha sido fechado en más de 12 millones de años (Israde-Alcántara et al., 2004). Pruebas de acuífero realizadas en la zona indican que la unidad volcánica se comporta como un acuífero confinado, mientras que la unidad terrígena tiene un carácter semiconfinado o libre.

\section{Metodología}

Se seleccionaron 35 sitios, que incluyen 29 pozos y 6 manantiales. Cada sitio fue muestreado durante el periodo de abril a junio del año 2015.

Para identificar los sistemas de flujo se consideraron tres parámetros, que incluyen la temperatura, los iones mayores y la profundidad del agua subterránea (nivel estático). Estos tres parámetros han sido señalados en diversos estudios donde se caracterizan los sistemas de flujo de agua subterránea (Carrillo-Rivera et al., 1996; Cardona, CarrilloRivera, 2006; Mahlknecht et al., 2006; CarrilloRivera et al., 2007; Fagundo-Castillo et al., 2014; Huizar-Alvarez et al., 2016). Tóth ilustra como los parámetros de temperatura, contenido de iones mayores y profundidad cambian en los sistemas de flujo conforme estos circulan y evolucionan en su naturaleza local, intermedia o regional (Tóth, 1999).

\subsection{MEDICIÓN DE LA TEMPERATURA}

La temperatura se determinó con un equipo 
marca Hanna modelo HI 9828 con una sonda HI 769828.

\subsection{TOMA, TRANSPORTE Y ALMAGENAMIENTO DE MUESTRA}

Las muestras se tomaron directamente de la válvula de salida del pozo, dejando correr el agua por 10 minutos antes de la colecta de agua subterránea. En los manantiales la muestra se tomó en el punto más cercano al afloramiento de agua subterránea. La muestra se filtró en una membrana de celulosa de 0.45 micras marca Millipore. Posteriormente se dividió en dos partes, una para aniones y la otra para cationes. Los cationes se acidificaron a $\mathrm{pH}$ de 2 usando ácido nítrico al $65.70 \%$ grado analítico, marca J.T. Baker. Cada muestra se colocó en envases de polietileno de alta densidad, fue sellada con papel parafilm (cera) y se transportó en una hielera hasta el laboratorio, donde se almacenaron a $4{ }^{\circ} \mathrm{C}$ hasta el momento de su análisis.

\subsection{ANÁLISIS DE ANIONES}

La alcalinidad (expresada como bicarbonato) se midió en campo. Se tomó una muestra de $20 \mathrm{ml}$, a la cual se le agregaron dos gotas de solución indicadora naranja de metilo. Posteriormente se tituló la muestra con ácido sulfúrico $1.6 \mathrm{~N}$ por medio de un equipo de titulación portátil marca HACH. Los cloruros y sulfatos se determinaron de acuerdo con el método EPA 300.1 en el Laboratorio de Geoquímica Ambiental del Centro de Geociencias de la Universidad Nacional Autónoma de México (UNAM). El análisis químico se llevó a cabo mediante un cromatógrafo de líquidos marca Dionex LC20 con supresor ASRS-Ultra 300 4-mm, columna Ion Pac AS14A y detector electroquímico ED50.

\subsection{ANÁLISIS DE GATIONES}

Los análisis de cationes mayores, menores y elementos traza fueron realizados de acuerdo con el método EPA 200.8, en el Laboratorio de Análisis Químicos del Departamento de Ingeniería
Metalúrgica de la Facultad de Química de la UNAM, usando un equipo de espectrometría de emisión óptica marca Perkin Elmer modelo ICPOES Optima serie 8300 DV.

\subsection{MEDIGIÓN DE LA PROFUNDIDAD DEL AGUA SUBTERRÁNEA}

La profundidad del agua en los pozos se midió a través de una sonda marca Solinst modelo Water Level Meter 101 con una longitud de $300 \mathrm{~m}$. Las mediciones del nivel estático se realizaron después de haber apagado la bomba, habiendo dejado descansar el pozo un mínimo de 12 horas. Para los manantiales la profundidad se consideró cero.

\section{Resultados y Discusión}

Los análisis químicos de las muestras de agua subterránea se presentan en la Tabla 1, en la que se observan muestras que sobrepasaron el $10 \%$ de error en el balance iónico, lo cual se asocia a un error en la medición del potasio. En la Tabla 2 se presentan los datos de temperatura, iones mayores y profundidad (nivel estático) del agua subterránea de los sitios de estudio. Los pozos presentaron valores promedio de temperatura de $24.92{ }^{\circ} \mathrm{C}$, con un valor mínimo de $21.00^{\circ} \mathrm{C}$ y un máximo de $31.17^{\circ} \mathrm{C}$, mientras que los iones mayores tuvieron una media de $252.19 \mathrm{mg} / \mathrm{l}$, con un mínimo de $125.38 \mathrm{mg} / \mathrm{l}$ y un máximo de $673.31 \mathrm{mg} / \mathrm{l}$. Los manantiales tuvieron una temperatura promedio de $22.67^{\circ} \mathrm{C}$, siendo el valor mínimo de $20.00^{\circ} \mathrm{C}$ y el máximo de $32.50{ }^{\circ} \mathrm{C}$. La media de iones mayores fue de $259.53 \mathrm{mg} / \mathrm{l}$, con un valor mínimo de $134.40 \mathrm{mg} / \mathrm{l}$ y un máximo de $411.27 \mathrm{mg} / \mathrm{l}$.

En el año 1994 el agua subterránea se encontraba a una profundidad promedio de $30 \mathrm{~m}$ y en el año 2015 el promedio en los pozos muestreados fue de $74.63 \mathrm{~m}$, por lo tanto se observa un abatimiento de $44.63 \mathrm{~m}$ que está directamente relacionado con la extracción del agua del subsuelo; considerando el periodo de tiempo (21 años), se tiene un abatimiento promedio de $2.13 \mathrm{~m} /$ año, dato similar al de $2.46 \mathrm{~m} /$ año reportado por Ávila-Olivera y 
Tabla 1. Balances iónicos de las muestras de agua. El asterisco indica las muestras que sobrepasaron el $15 \%$ de error en el balance iónico.

\begin{tabular}{|c|c|c|c|c|c|c|c|c|c|c|c|c|c|c|c|}
\hline ID & $F^{-}$ & $\mathrm{Cl}^{-}$ & $\mathrm{NO}_{2}^{-}$ & $\mathrm{Br}^{-}$ & $\mathrm{NO}_{3}^{-}$ & $\mathrm{PO}_{4}{ }^{3-}$ & $\mathrm{SO}_{4}{ }^{2-}$ & $\mathrm{HCO}_{3}^{-}$ & $\mathrm{Ca}^{2+}$ & $\mathrm{Mg}^{2+}$ & $\mathbf{K}^{+}$ & $\mathrm{Na}^{+}$ & Iones & Iones & Balance \\
\hline art & meq & meq & meq & meq & meq & meq & meq & meq & meq & meq & meq & meq & - & + & $\%$ \\
\hline $\mathrm{P} 1$ & 0.01 & 0.89 & 0.01 & 0 & 0.03 & 0 & 0.14 & 2.97 & 0.78 & 0.49 & 0.01 & 1.94 & 4.04 & 3.22 & -11.27 \\
\hline P2 & 0.01 & 0.48 & 0 & 0 & 0.05 & 0 & 0.09 & 1.44 & 0.21 & 0.15 & 0.01 & 0.43 & 2.07 & 0.8 & $-44.14 *$ \\
\hline P3 & 0.02 & 0.1 & 0 & 0 & 0.07 & 0 & 0.05 & 1.8 & 0.39 & 0.3 & 0.01 & 0.94 & 2.04 & 1.64 & -10.74 \\
\hline P4 & 0.01 & 0.19 & 0 & 0 & 0.07 & 0 & 0.08 & 1.98 & 0.56 & 0.48 & 0.02 & 1.03 & 2.33 & 2.09 & -5.38 \\
\hline P5 & 0.01 & 0.08 & 0 & 0 & 0.07 & 0 & 0.04 & 2.61 & 0.57 & 0.9 & 0.02 & 0.79 & 2.81 & 2.27 & -10.53 \\
\hline P6 & 0.01 & 1.31 & 0.01 & 0 & 0.01 & 0 & 0.28 & 6.48 & 1.61 & 0.97 & 0.02 & 3.2 & 8.11 & 5.8 & $-16.59^{*}$ \\
\hline P7 & 0.01 & 0.38 & 0 & 0 & 0.03 & 0 & 0.4 & 4.32 & 1.19 & 1.42 & 0.03 & 1.18 & 5.14 & 3.83 & -14.64 \\
\hline P8 & 0.01 & 0.08 & 0 & 0 & 0.04 & 0 & 0.09 & 2.61 & 0.73 & 0.66 & 0.02 & 0.95 & 2.83 & 2.36 & -9.13 \\
\hline P9 & 0.01 & 2.96 & 0.02 & 0 & 0.03 & 0 & 0.29 & 6.75 & 1.43 & 0.63 & 0.02 & 4.59 & 10.05 & 6.67 & $-20.24 *$ \\
\hline P10 & 0.01 & 1.91 & 0.01 & 0 & 0.01 & 0 & 0.28 & 5.58 & 1.32 & 0.49 & 0.02 & 3.84 & 7.8 & 5.66 & $-15.92^{*}$ \\
\hline P11 & 0.01 & 0.28 & 0 & 0 & 0.14 & 0 & 0.08 & 3.15 & 0.72 & 1.14 & 0.02 & 1.1 & 3.65 & 2.98 & -10.16 \\
\hline P12 & 0.01 & 0.1 & 0 & 0 & 0.03 & 0.01 & 0.03 & 2.88 & 0.39 & 0.61 & 0.02 & 1.16 & 3.07 & 2.18 & $-16.94 *$ \\
\hline P13 & 0.01 & 0.11 & 0 & 0 & 0.09 & 0 & 0.06 & 1.89 & 0.57 & 0.86 & 0.02 & 0.79 & 2.16 & 2.24 & 1.84 \\
\hline P14 & 0.01 & 0.09 & 0 & 0 & 0.06 & 0 & 0.04 & 1.89 & 0.46 & 0.71 & 0.02 & 0.91 & 2.1 & 2.11 & 0.3 \\
\hline P15 & 0.01 & 0.05 & 0 & 0 & 0.05 & 0 & 0.04 & 2.61 & 0.82 & 0.96 & 0.02 & 0.93 & 2.76 & 2.72 & -0.7 \\
\hline P16 & 0.01 & 0.07 & 0 & 0 & 0.1 & 0 & 0.05 & 2.07 & 0.56 & 0.43 & 0.02 & 0.86 & 2.3 & 1.88 & -10.08 \\
\hline P17 & 0.01 & 0.87 & 0.01 & 0 & 0.28 & 0 & 0.33 & 2.07 & 0.56 & 0.84 & 0.01 & 1.97 & 3.56 & 3.39 & -2.52 \\
\hline P18 & 0.01 & 0.15 & 0 & 0 & 0.13 & 0 & 0.09 & 2.16 & 0.63 & 0.83 & 0.02 & 0.84 & 2.54 & 2.32 & -4.58 \\
\hline P19 & 0.01 & 0.09 & 0 & 0 & 0.13 & 0 & 0.08 & 2.07 & 0.65 & 0.74 & 0.02 & 0.64 & 2.37 & 2.05 & -7.26 \\
\hline P20 & 0.02 & 0.06 & 0 & 0 & 0.02 & 0.02 & 0.16 & 2.34 & 0.46 & 0.79 & 0.02 & 0.84 & 2.61 & 2.11 & -10.64 \\
\hline $\mathrm{P} 21$ & 0.01 & 0.07 & 0 & 0 & 0 & 0 & 0.06 & 3.24 & 0.59 & 1.09 & 0.02 & 1.33 & 3.37 & 3.04 & -5.23 \\
\hline P22 & 0.01 & 0.12 & 0 & 0 & 0.11 & 0 & 0.07 & 2.25 & 0.57 & 0.86 & 0.02 & 0.85 & 2.56 & 2.31 & -5.05 \\
\hline P23 & 0.01 & 0.1 & 0 & 0 & 0.03 & 0 & 0.04 & 2.43 & 0.43 & 0.54 & 0.02 & 0.97 & 2.61 & 1.96 & -14.16 \\
\hline P24 & 0.01 & 0.12 & 0 & 0 & 0.08 & 0 & 0.04 & 2.16 & 0.53 & 0.86 & 0.02 & 0.84 & 2.41 & 2.25 & -3.27 \\
\hline P25 & 0.01 & 2.14 & 0 & 0 & 0 & 0 & 0.31 & 6.12 & 1.01 & 0.84 & 0.02 & 4.73 & 8.59 & 6.6 & -13.1 \\
\hline M1 & 0.01 & 0.25 & 0 & 0 & 0.21 & 0 & 0.15 & 1.44 & 0.53 & 0.79 & 0.01 & 0.43 & 2.05 & 1.76 & -7.71 \\
\hline P26 & 0.01 & 0.16 & 0 & 0 & 0.21 & 0 & 0.13 & 1.44 & 0.58 & 0.85 & 0.01 & 0.51 & 1.94 & 1.95 & 0.18 \\
\hline P27 & 0.01 & 0.12 & 0 & 0 & 0 & 0 & 0.1 & 2.43 & 0.58 & 0.87 & 0.02 & 1.01 & 2.66 & 2.49 & -3.38 \\
\hline M2 & 0 & 0.05 & 0 & 0 & 0 & 0 & 0.02 & 5.22 & 1.5 & 1.63 & 0.02 & 1.71 & 5.29 & 4.86 & -4.22 \\
\hline P28 & 0.01 & 0.06 & 0 & 0 & 0.06 & 0 & 0.03 & 1.53 & 0.4 & 0.6 & 0.01 & 0.64 & 1.69 & 1.65 & -1.15 \\
\hline P29 & 0.01 & 0.08 & 0 & 0 & 0.27 & 0 & 0.14 & 1.71 & 0.56 & 0.78 & 0.01 & 0.53 & 2.2 & 1.88 & -7.92 \\
\hline M3 & 0.01 & 0.27 & 0 & 0 & 0.34 & 0 & 0.33 & 2.43 & 1.08 & 1.35 & 0.02 & 1.23 & 3.37 & 3.69 & 4.48 \\
\hline M4 & 0.01 & 0.75 & 0 & 0 & 0.53 & 0 & 0.54 & 2.52 & 0.86 & 1.12 & 0.01 & 1.66 & 4.36 & 3.65 & -8.78 \\
\hline M5 & 0.01 & 0.43 & 0 & 0 & 0.97 & 0 & 0.8 & 1.98 & 0.98 & 1.31 & 0.01 & 0.93 & 4.2 & 3.22 & -13.11 \\
\hline M6 & 0.01 & 0.5 & 0 & 0 & 1.06 & 0 & 0.98 & 2.16 & 1.39 & 1.57 & 0.02 & 0.81 & 4.71 & 3.79 & -10.84 \\
\hline
\end{tabular}

Garduño-Monroy (2007) en la ciudad de Morelia. Los abatimientos medidos en el año 2015 presentaron valores diversos; algunos sitios se encuentran en valores similares a los reportados en el año 1994 (P7, P8, P23, P27), mientras que otros superan los 100 m (P1, P2, P3, P4, P10, P12, P13, P14), lo que da una idea de la heterogeneidad de las propiedades hidrogeológicas y de los esquemas de extracción de agua subterránea de la zona, así como de la construcción y mantenimiento de los pozos. El caso de Morelia-Capula pone en evidencia que la extracción de agua subterránea puede generar importantes cambios en los niveles piezométricos, y con ello en la dinámica de los sistemas de flujo, en periodos de tiempo relativamente cortos, considerando que la recarga natural de un acuífero puede tardar cientos de años (Gleeson et al., 2010), décadas cuando los flujos son locales y siglos cuando los flujos son regionales. Además, el abatimiento de los niveles piezométricos puede implicar un riesgo para la calidad del agua. Estudios previos han demostrado que a mayor profundidad se localice el recorrido del agua subterránea, existe un incremento de las especies químicas indeseables para la salud humana (Cardona y Carrillo-Rivera, 2006; Huizar-Alvarez et al., 2004). 
La superficie piezométrica, los diagramas de Stiff, la temperatura y el comportamiento espacial de los sistemas de flujo se muestran en la Figura 2. La superficie piezométrica ilustra que en general la tendencia de flujo de agua subterránea de la zona es de oeste a este. Los diagramas de Stiff indican que el contenido salino es menor en las faldas del cerro del Águila y los volcanes Quinceo y Las Tetillas, evidencia de que estas zonas son de recarga. En la Figura 3 se muestra la gráfica de temperatura-iones mayores de los sitios de estudio; se observan tres agrupamientos, el primero, asociado a flujos locales, se ubica entre 20.00 y $21.00{ }^{\circ} \mathrm{C}$ y 133.85 y $275.94 \mathrm{mg} / \mathrm{l}$, el segundo, correspondiente al sistema intermedio, se ubica entre 21.50 y $31.17^{\circ} \mathrm{C}$ y 127.30 y $365.41 \mathrm{mg} / \mathrm{l}$ y el tercero, de carácter regional, se ubica entre 25.50 y $32.50{ }^{\circ} \mathrm{C}$ y 411.27 y $673.31 \mathrm{mg} / \mathrm{l}$. Algunos pozos presentan agua con características intermedias entre sistemas de flujo local-intermedio (P23, P27) y otros, intermedio-regional (P6, P9, P25). De los treinta y cinco sitios de estudio, ocho se aso- ciaron a flujo local, veintiséis a intermedio y uno a regional (Tabla 2). En los manantiales, cinco sitios presentan flujos de carácter local y uno de carácter regional (M2). En los pozos, tres sitios (P23, P26, P27) presentan carácter local, los demás como intermedios y algunos de ellos con tendencia a regional (P6, P9, P10 y $\mathrm{P} 25)$.

El hecho de que en los pozos se hayan encontrado tanto flujos locales como intermedios e intermedios-regionales se debe a que estos fueron construidos a diferentes profundidades sin considerar criterios hidrogeológicos. Además, cuando los pozos fueron construidos se instaló tubería ranurada desde la profundidad donde se encontró agua subterránea (aproximadamente a $30 \mathrm{~m}$ de profundidad en el año de 1994) hasta su extremo inferior, con lo cual se permitió la mezcla de flujos de diferentes profundidades. En los sitios P25 y M2, ubicados directamente sobre fallas geológicas, se observó que estas conectan flujos profundos con la superficie, hecho que quedó evidenciado en el incremento de la temperatura y la cantidad de

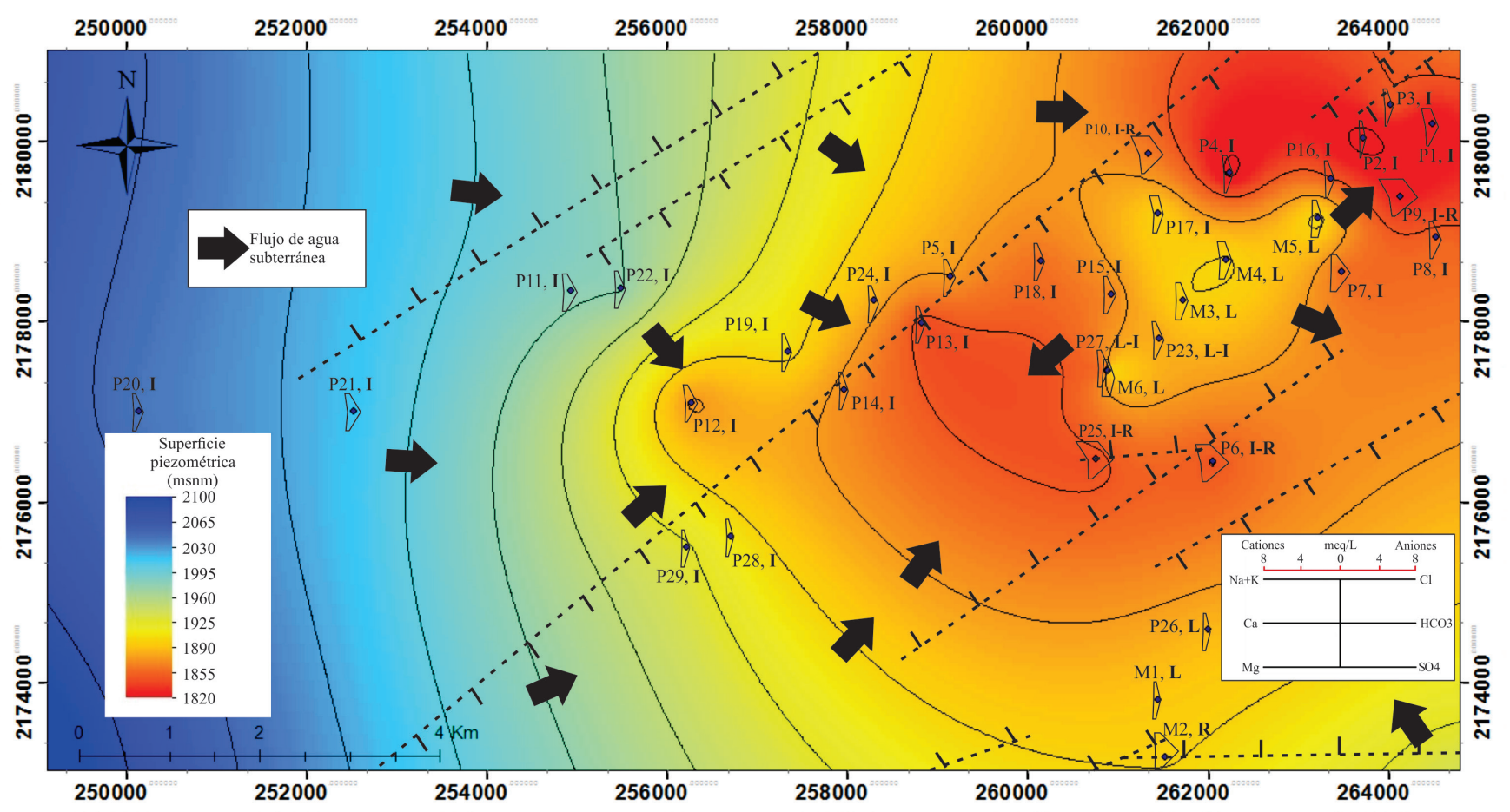

Figura 2 Comportamiento general del flujo de agua subterránea en el plano horizontal. Se indican los diagramas de Stiff, la distribución de la superficie piezométrica y el tipo de flujo local (L), intermedio (I) y regional (R). 


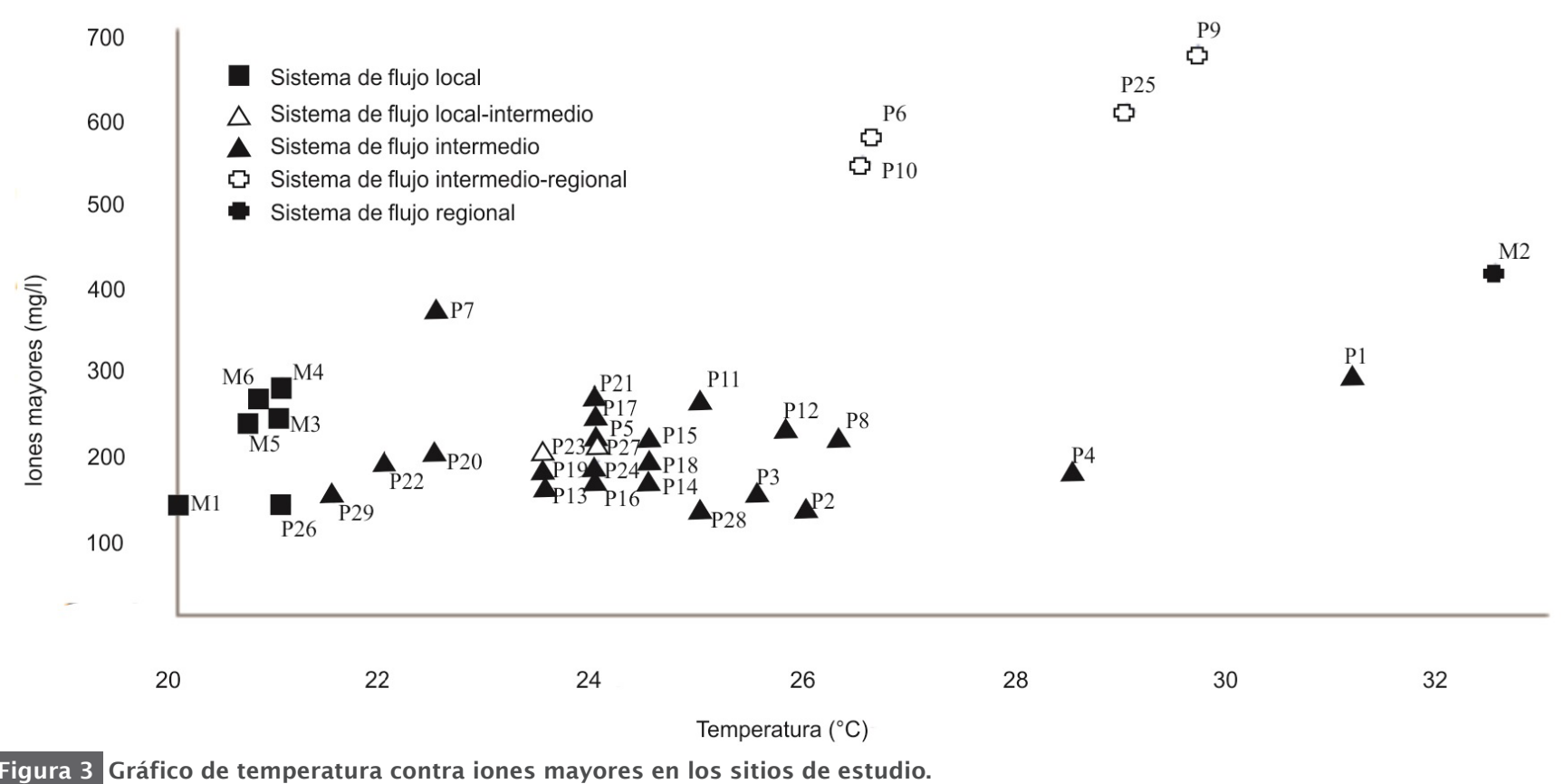

Figura 3 Gráfico de temperatura contra iones mayores en los sitios de estudio.

iones mayores en el agua subterránea. La conexión de diferentes sistemas de flujo por medio de fallas geológicas quedó evidenciado en la parte sureste de la zona de estudio, ya que ahí existen pozos (P6 y P25) y un manantial (M6), ubicados directamente sobre las fallas que presentan valores altos de temperatura y de iones mayores.

En la Figura 4 se muestran secciones geológicas donde se indica las direcciones de flujo de los diferentes sistemas. En ella se observa que la tendencia general de flujo va de oeste a este y que las partes altas de la zona (complejo volcánico El Quinceo-Las Tetillas y el Cerro del Águila) favorecen el flujo subterráneo de los sistemas local e intermedio hacia las partes bajas donde se ubican los manantiales. Debido a que los incrementos de temperatura y de iones mayores se observaron sólo en los sitios ubicados sobre fallas geológicas, se infiere que la dirección de flujo del sistema regional es de tipo vertical ascendente. La información generada permite conceptualizar la zona MoreliaCapula como un sistema acuífero con flujos de jerarquía local, intermedia y regional que están interconectados hidráulicamente. En la Figura 5 se presenta un modelo conceptual del sistema de flujos presentes en la zona Morelia-Capula.

\subsection{SISTEMA DE FLUJO LOGAL}

El sistema de flujo local se encuentra en manantiales y pozos poco profundos. Los sitios de estudio asociados a flujos locales se caracterizan por una temperatura promedio de $21.50{ }^{\circ} \mathrm{C}$ (mínimo $20.00{ }^{\circ} \mathrm{C}$ y máximo $24.00{ }^{\circ} \mathrm{C}$ ), iones mayores de $209.43 \mathrm{mg} / \mathrm{l}$ (mínimo $133.85 \mathrm{mg} / \mathrm{l}$ y máximo $275.94 \mathrm{mg} / \mathrm{l}$ ) y nivel estático en los pozos a una profundidad de $21.84 \mathrm{~m}$ (mínimo $5 \mathrm{~m}$ y máximo $38.38 \mathrm{~m})$. El flujo local se mueve en los estratos superiores del sistema acuífero tanto en la unidad volcánica como en la terrígena. El flujo local genera manantiales que brotan en los puntos de contacto entre materiales volcánicos y fluviolacustres, conformados por estratos inter digitados de arcillas y arenas. La presencia de manantiales en los contactos litológicos de los materiales fluviolacustres se asocia a que esta unidad hidrogeológica se ubica en la parte más baja de la zona de estudio permitiendo el tránsito de flujos locales. Debido a su corto recorrido los flujos locales son susceptibles 
Tabla 2. Parámetros y clasificación de los flujos de la zona Morelia-Capula.

\begin{tabular}{|c|c|c|c|c|}
\hline ID & $\begin{array}{c}\mathrm{T} \\
\left({ }^{\circ} \mathrm{C}\right)\end{array}$ & $\begin{array}{c}\text { Iones mayores } \\
(\mathrm{mg} / \mathrm{l})\end{array}$ & $\begin{array}{l}\text { Profundidad } \\
\text { (m) }\end{array}$ & Flujo \\
\hline M1 & 20 & 134.4 & 0 & Local \\
\hline M3 & 21 & 240.81 & 0 & Local \\
\hline M4 & 21 & 275.94 & 0 & Local \\
\hline M5 & 20.7 & 232.05 & 0 & Local \\
\hline M6 & 20.82 & 262.73 & 0 & Local \\
\hline P26 & 21 & 133.85 & 5 & Local \\
\hline $\mathrm{P} 23$ & 23.5 & 191.97 & 22.15 & Local (con tendencia a intermedio) \\
\hline $\mathrm{P} 27$ & 24 & 203.67 & 38.38 & Local (con tendencia a intermedio) \\
\hline $\mathrm{P} 1$ & 31.17 & 285.91 & 123 & Intermedio \\
\hline $\mathrm{P} 2$ & 26 & 125.38 & 137.9 & Intermedio \\
\hline $\mathrm{P} 3$ & 25.5 & 149.44 & 116.4 & Intermedio \\
\hline P4 & 28.5 & 172.69 & 123.5 & Intermedio \\
\hline P5 & 24 & 205.1 & 83.28 & Intermedio \\
\hline P7 & 22.5 & 365.41 & 31 & Intermedio \\
\hline P8 & 26.3 & 211.83 & 39.21 & Intermedio \\
\hline P11 & 25 & 260.25 & 51.7 & Intermedio \\
\hline P12 & 25.8 & 223.5 & 135 & Intermedio \\
\hline $\mathrm{P} 13$ & 23.5 & 162.71 & 103.1 & Intermedio \\
\hline P14 & 24.5 & 160.22 & 100 & Intermedio \\
\hline P15 & 24.5 & 213.24 & 59.67 & Intermedio \\
\hline P16 & 24 & 168.06 & 69.27 & Intermedio \\
\hline P17 & 24 & 239.89 & 42.38 & Intermedio \\
\hline P18 & 24.5 & 184.33 & 98.83 & Intermedio \\
\hline P19 & 23.5 & 170.6 & 63 & Intermedio \\
\hline P20 & 22.5 & 191.67 & 49.33 & Intermedio \\
\hline $\mathrm{P} 21$ & 24 & 259.23 & 68 & Intermedio \\
\hline $\mathrm{P} 22$ & 22 & 187.24 & 49.71 & Intermedio \\
\hline $\mathrm{P} 24$ & 24 & 178.9 & 69.75 & Intermedio \\
\hline P28 & 25 & 127.3 & 72 & Intermedio \\
\hline P29 & 21.5 & 147 & 68 & Intermedio \\
\hline P6 & 26.6 & 573.81 & 58.73 & Intermedio (con tendencia a regional) \\
\hline P9 & 29.7 & 673.31 & 83.28 & Intermedio (con tendencia a regional) \\
\hline P10 & 26.5 & 542.82 & 141.03 & Intermedio (con tendencia a regional) \\
\hline P25 & 29 & 604.16 & 61.8 & Intermedio (con tendencia a regional) \\
\hline M2 & 32.5 & 411.27 & 0 & Regional \\
\hline
\end{tabular}



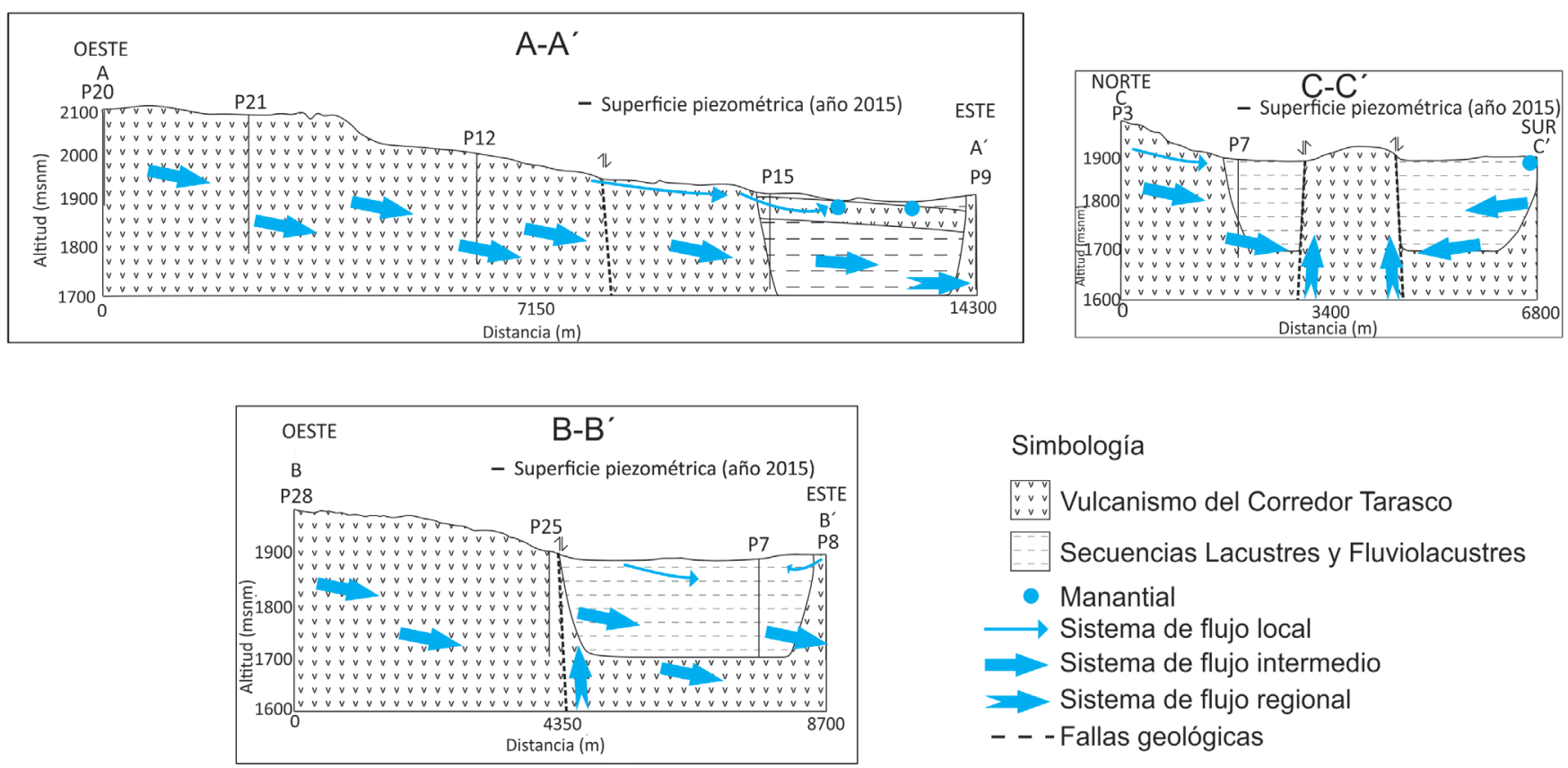

Figura 4 Dinámica de los sistemas de flujo en secciones geológicas de la zona Morelia-Capula.

a la contaminación antropogénica debido a que en la zona existe descarga de agua residual, además puede haber percolación de agroquímicos en las zonas de cultivo (de temporal).

\subsection{SISTEMA DE FLUJO INTERMEDIO}

Los sitios pertenecientes al sistema de flujo intermedio presentan una temperatura promedio de $25.39{ }^{\circ} \mathrm{C}$ (mínimo $21.50{ }^{\circ} \mathrm{C}$ y máximo $32.50{ }^{\circ} \mathrm{C}$ ), iones mayores de $255.86 \mathrm{mg} / \mathrm{l}$ (mínimo 125.38 mg/l y máximo $673.71 \mathrm{mg} / \mathrm{l}$ ) y nivel estático en los pozos a una profundidad de $75.30 \mathrm{~m}$ (mínimo 31 m y máximo 137.90 m). Los sistemas de flujos intermedios se encuentran por toda la zona de estudio, subyaciendo a los locales. Los sistemas de flujo intermedio se encuentran tanto en la unidad volcánica como en la terrígena. En algunos pozos (P7，P8，P17，P19, P20, P22, P29) los sistemas de flujo intermedios entran en contacto con los sistemas locales; estos sitios se caracterizan por tener temperatura y (o) iones mayores cercanos al promedio de los flujos locales. Los sitios P6, P9, P10 y P25 son flujos intermedios con tendencia a regional, debido a su alto contenido salino y su temperatura. La mayoría de los aprovechamientos subterráneos de la zona están extrayendo agua de flujos intermedios. Es de esperar que en los años venideros el descenso de los niveles piezométricos ocasione que los pozos se construyan a más de 350 $\mathrm{m}$ de profundidad y se recurra a flujos regionales para satisfacer la necesidad de agua de la zona. Este sistema de flujo puede considerarse como sujeto a una extracción intensiva, debido al abatimiento continuo de los niveles piezométricos, ocasionado por los bombeos.

\subsection{SISTEMA DE FLUJO REGIONAL}

La presencia de flujos regionales se evidencia en el sitio M2. Su temperatura es $32.50{ }^{\circ} \mathrm{C}$ y su contenido de iones mayores es $411.27 \mathrm{mg} / \mathrm{l}$. Algunos pozos entran en contacto con el sistema de flujo regional, tal es el caso del P10, el cual está construido a una profundidad de $350 \mathrm{~m}$, además de estar sujeto a un régimen de operación de 20 horas diarias, por lo cual se infiere que estos factores (profundidad y horas de bombeo) han ocasionado que los flujos locales e intermedios se hayan agotado y se esté extrayendo agua de flujos más profundos 


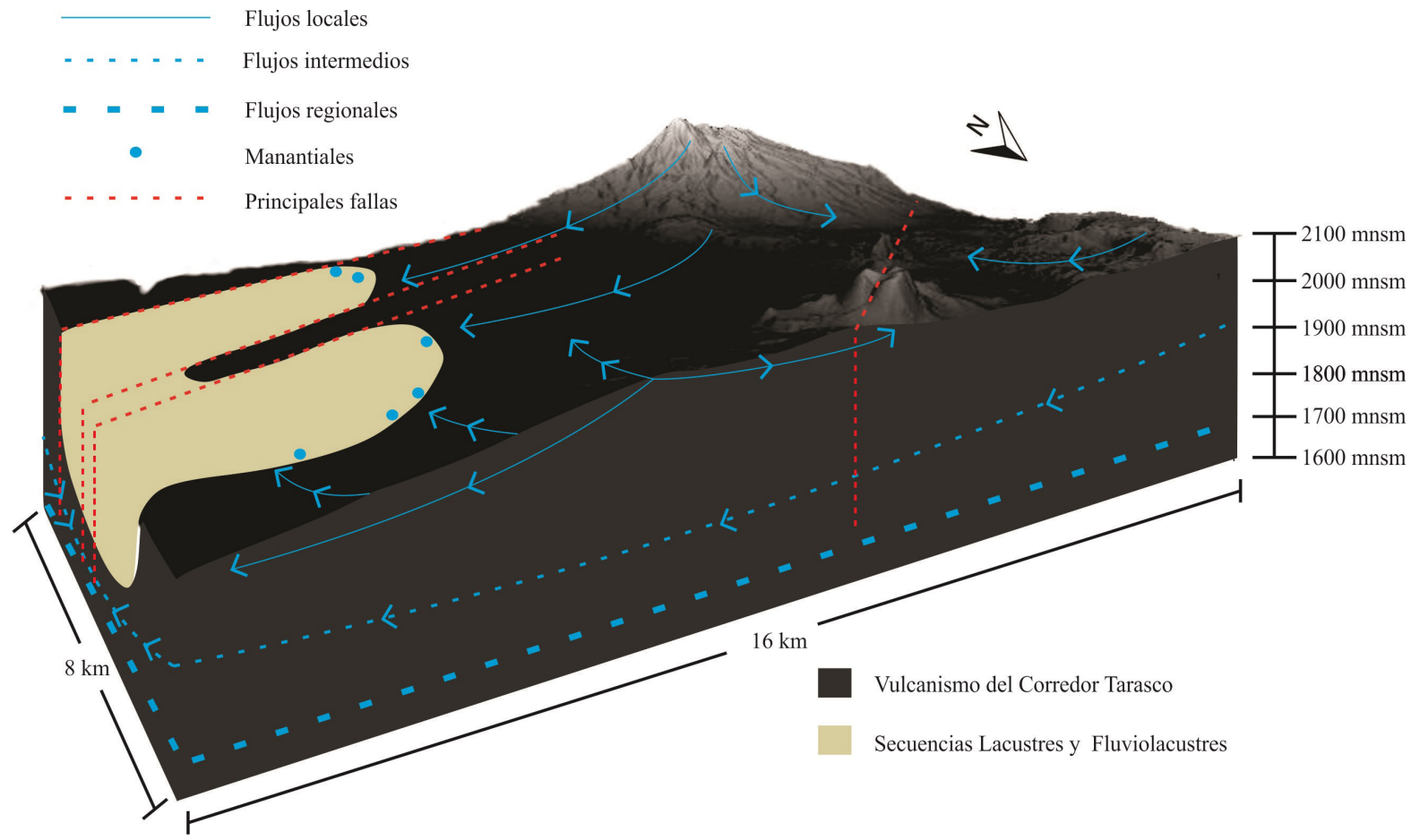

Figura 5 Sistemas de flujo de agua subterránea de la zona Morelia-Capula.

de jerarquía intermedia-regional. El sistema de flujo regional se encuentra en la unidad volcánica, la cual subyace a la terrígena. La información obtenida apunta a que este sistema tiene dirección vertical ascendente.

En la zona Morelia-Capula, los límites de contacto entre materiales volcánicos y fluviolacustres están definidos por las fallas geológicas, por lo que la geología estructural está jugando un papel central en la dinámica de los sistemas de flujo de agua subterránea, lo que genera zonas de afloramiento de manantiales o actuando como canales de interconexión de flujos profundos con superficiales.

El modelo conceptual muestra que en forma general los sistemas de flujo de la zona Morelia-Capula siguen la dinámica natural establecida por la topografía del terreno, aunque existen afectaciones puntuales (P1, P2, P4, P12, P13) donde los flujos de agua subterránea han invertido sus direcciones debido a los conos de abatimiento ocasionados por la extracción intensiva.

El reconocimiento de los sistemas de flujo de la zona Morelia-Capula provee ciertas directrices a considerar para el manejo del agua subterránea.
Tal como es señalado en las referencias consultadas, el tipo de materiales geológicos, el clima y la topografía juegan papeles centrales para determinar la dinámica y los tipos de flujo presentes en una determinada zona de estudio (Sanford, 2002; Zhou y Li, 2011). Estas consideraciones deben ser tomadas en cuenta para el manejo del agua subterránea. En el caso de la zona Morelia-Capula, aunque las lluvias no son abundantes, sí permiten la generación de manantiales gracias a la presencia de materiales rocosos que permiten la infiltración, canalizándola en flujos locales que brotan en las partes bajas. Los flujos locales asociados a la infiltración de agua de lluvia representan un elemento valioso con carácter renovable. Es importante que se tenga un adecuado manejo de residuos sólidos y aguas residuales para evitar contaminar los sistemas de flujo locales. Algunas muestras presentaron contenidos altos de nitratos (M3, M4, M5, M6, P17 y P29), indicativo de que aguas residuales o agroquímicos pudieran estar entrando en contacto con los sistemas de flujo local e intermedio. Para dimensionar la importancia de los flujos locales, el manantial M1 provee alrededor de 500 litros por 
segundo, aproximadamente el $13.60 \%$ del agua que se consume en Morelia. Los datos muestran la importancia en el suministro de agua de los sistemas locales e intermedios, sin embargo, se desconoce su volumen de recarga. La mayor parte de los pozos se abastecen del sistema de flujo intermedio e intermedio-regional. Estos sistemas pueden ser considerados no renovables (a corto y mediano plazo) ya que la edad promedio del agua subterránea puede ser de varios cientos de años (Mays, 2013). Para establecer un equilibrio en el sistema de flujo intermedio-regional se recomienda una estrategia enfocada a optimizar el uso del agua, evitando pérdidas en la red de distribución, además de aprovechar el agua de lluvia y tratar las aguas residuales a nivel de agua potable pensando en su posible reinyección al sistema acuífero, previo estudios de factibilidad correspondiente.

\section{Conclusiones y Recomendaciones}

En el subsuelo de la zona Morelia-Capula se encuentra un sistema acuífero donde interactúan flujos de jerarquía local, intermedia y regional. La mayor parte de los pozos se abastecen del sistema de flujo intermedio, adicionalmente los pozos presentan evidencias de mezcla con flujo local y regional. Las mezclas de flujos se ven favorecidas por las características de construcción de los pozos (ranurado a todo lo largo) y por las fallas geológicas presentes en la zona. En el plano horizontal los sistemas de flujo local e intermedio se mueven siguiendo la topografía de la zona (de oeste a este), aunque en forma puntual el abatimiento en los pozos de extracción ha ocasionado cambio en la dirección natural de flujo, mientras que el sistema de flujo regional presenta una dirección ascendente vertical. En los estratos superficiales del sistema acuífero el agua subterránea es vulnerable a la contaminación debido a la presencia de flujos locales. Es necesario implementar estrategias enfocadas a disminuir la presión hídrica sobre el sistema de flujo intermedio, de lo contrario se continuará la tendencia de abatimiento de los niveles piezométricos del sistema acuífero y con ello a la extracción de flujo regional.

Los resultados obtenidos a partir del estudio de la zona Morelia-Capula desde la perspectiva de los sistemas de flujo permiten generar conocimiento para mejorar el manejo del agua subterránea, que es una información útil para usuarios y administradores del agua subterránea. Es necesario profundizar en el estudio del funcionamiento de los sistemas de flujo de agua subterránea de todo el país. Para tal tarea es fundamental involucrar a las administraciones del agua en sus diferentes niveles. Así sería posible pasar de esquemas de extracción y suministro a esquemas enfocados a preservar las aguas subterráneas, basándose en el conocimiento de los sistemas de flujo.

\section{Agradecimientos}

Agradecemos a la M. en C. Carolina Muñoz Torres, del laboratorio de Geoquímica Ambiental del Centro de Geociencias de la UNAM, por haber realizado los análisis de aniones. Igualmente agradecemos al Dr. Ciro Eliseo Márquez Herrera, por haber facilitado las instalaciones y equipos del Laboratorio de Análisis Químicos del Departamento de Ingeniería Metalúrgica de la Facultad de Química de la UNAM, para realizar el análisis de cationes. Se desea hacer patente nuestro agradecimiento a la Comisión Local (OOAPAS) y Comisión Nacional del Agua (CONAGUA) por permitirnos el acceso a sus bases de datos para recopilar información de la zona Morelia-Capula.

\section{Referencias}

Ávila-Olivera, J.A., Garduño-Monroy, V.H., 2007, Análisis del abatimiento de los niveles freáticos del sistema acuífero de Morelia: Ciencia Nicolaita, 46, 105-122.

Cardona, A., Carrillo-Rivera, J.J., 2006, Hidrogeoquímica de sistemas de flujo intermedio que circulan por sedimentos 
continentales derivados de rocas riolíticas: Ingeniería Hidráulica en México, 21(3), 69-86.

Carlón-Allende, T., Mendoza, M.E., 2007, Análisis hidrometeorológico de las estaciones de la cuenca del lago de Cuitzeo: Investigaciones Geográficas, 63, 56-76.

Carreón-Freyre, D., Cerca, M., Luna-González, L., Gámez-González, FJ., 2005, Influencia de la estratigrafía y estructura geológica en el flujo de agua subterránea del valle de Querétaro: Revista Mexicana de Ciencias Geológicas, 22(1), 1-18.

Carrillo-Rivera, J.J., Cardona, A., 2012, Groundwater flow systems and their response to climate change: A need for a watersystem view approach, American Journal of Environmental Sciences, 8(3), 220-35.

Carrillo-Rivera, J.J., Cardona, A., Moss, D., 1996, Importance of the vertical component of groundwater flow: A hydrogeochemical approach in the valley of San Luis Potosi, Mexico: Journal of Hydrology, 185, 23-44.

Carrillo-Rivera, J.J., Varsányi, I., Kovács, L.O., Cardona, A., 2007, Tracing groundwater flow systems with hydrogeochemistry in contrasting geological environments: Water, Air, and Soil Pollution, 184(1-4), 77-103.

Cisneros-Máximo, G., 2016, Geomorfología volcánica de Morelia y alrededores. Propuesta de cartografía geomorfológica jerárquica a nivel detalle y semidetalle: Universidad Nacional Autónoma de México, Morelia, México, Tesis de maestría, $150 \mathrm{p}$.

Comisión Nacional del Agua (CONAGUA), 2015, Estadísticas del agua en México: México, Secretaría del Medio Ambiente y Recursos Naturales, $58 \mathrm{p}$.

Dávila-Pórcel, R.A., de León-Gómez, H., 2011, Importancia de la hidrogeología urbana; ciencia clave para el desarrollo urbano sostenible: Boletín de La Sociedad Geológica Mexicana, 63(3), 463-477.

Environmental Protection Agency (EPA), 1994, Method 200.8, Determination of trace elements in waters and wastes by inductively coupled plasma-mass spectrometry, $57 \mathrm{p}$.

Environmental Protection Agency (EPA), 1999, Method 300.1, Determination of inorganic anions in drinking water by ion chromatography, $40 \mathrm{p}$.

Fagundo-Castillo, J.R., Alconada-Magliano, M.M., Garrillo-Rivera, J.J., GonzálezHernández, P., 2014, Caracterización de los flujos de agua subterránea a partir de su salinidad: Tecnología y Ciencias del Agua, 5(3), 63-80.

Garduño-Monroy, V.H., Medina-Vega V.H., Israde-Alcántara, I., Hernández-Madrigal, V.M., Ávila-Olivera, J.A., 2010, Unidades Geohidrológicas de la Región de MoreliaCuitzeo, Características Físicas, en Cram, S., Galicia, L., Israde-Alcántara, I. (comps.), Atlas de la cuenca del lago de Cuitzeo: Análisis de su geografía y entorno socio ambiental: México, UNAM, 66-69.

Garduño-Monroy, V.H., Giordano, N., ÁvilaOlivera, J.A., Hernández-Madrigal, V.M., Sámano-Nateras, A., Díaz-Salmerón, J.E., 2014, Estudio hidrogeológico del sistema acuífero de Morelia, Michoacán, para una correcta planificación del territorio, en Vieyra, A., Larraábal, A. (coords.), Urbanización, sociedad y medio ambiente. Experiencias en ciudades medias: México, UNAM/CIGA, SEMARNAT/INEGG, 197-222.

Gleeson, T., VanderSteen, J., Sophocleous, M.A., Taniguchi, M., Alley, W.M., Allen, D.M., Zhou, Y., 2010, Groundwater sustainability strategies, Nature Geoscience, 3(6), 378-379.

Huizar-Alvarez, R., Carrillo-Rivera, J.J., ÁngelesSerrano, G., Hergt, T., Cardona, A., 2004, Chemical response to groundwater extraction southeast of Mexico City, Hydrogeology Journal, 12(4), 436-450.

Huizar-Alvarez, R., Ouysse, S., EspinozaJaramillo, M.M, Carrillo-Rivera, J.J., Mendoza-Archundia, E., 2016, The effects of water use on Tothian flow systems in the Mexico City conurbation determined from 
the geochemical and isotopic characteristics of groundwater, Environmental Earth Sciences, 75(13), 2-17.

Israde-Alcántara, I., Garduño-Monroy, V.H., Ibañez-Garduño, D., Alatorre, M.A., Carrillo-Chávez, A., Agostino, R., Soto, L., 2004, Caracterización geológico ambiental del tiradero de Morelia y su relación con las poblaciones del entorno, en GarduñoMonroy, V.H. (ed.), Contribuciones a la geología e impacto ambiental de la Región de Morelia: Morelia, Universidad Michoacana de San Nicolás de Hidalgo, 137-155.

Israde-Alcántara, I., Buenrostro-Delgado, O., Carrillo-Chávez, A., 2005, Geological characterization and environmental implications of the placement of the Morelia dump, Michoacan, central Mexico: Journal of the Air \& Waste Management Association, 55(6), 755-764.

Mahlknecht, J., Gárfias-Solis, J., Aravena, R., Tesch, R., 2006, Geochemical and isotopic investigations on groundwater residence time and flow in the Independence Basin, Mexico: Journal of Hydrology, 324(1-4), 283-300.

Mays, L.W., 2013, Groundwater resources sustainability: Past, present, and future: Water
Resources Management, 27(13), 4409-4424. Ochoa-González, G.H., Carreón-Freyre, D., Cerca, M., López-Martínez, M., 2015, Assessment of groundwater flow in volcanic faulted areas. A study case in Queretaro, Mexico: Geofísica Internacional, 54(3), 199-220.

Peñuela-Arévalo, L.A., Carrilo-Rivera, J.J., 2013, Definición de zonas de recarga y descarga de agua subterránea a partir de indicadores superficiales: Centro-sur de la Mesa Central, México: Investigaciones Geográficas, Boletín del Instituto de Geografía, 81, 18-32.

Sanford, W., 2002, Recharge and groundwater models: an overview: Hydrogeology Journal, 10(1), 110-120.

Tóth, J., 1963, A theoretical analysis of groundwater flow in small drainage basins: Journal of Geophysical Research, 68(16), 4785-4812.

Tóth, J., 1999, Groundwater as a geologic agent: An overview of the causes, processes, and manifestations: Hydrogeology Journal, 7(1), $1-14$.

Zhou, Y., Li, W., 2011, A review of regional groundwater flow modeling: Geoscience Frontiers, 2(2), 205-214. 